\title{
Alocação de modelos de produtos a equipes de trabalhadores baseada em modelos de curvas de aprendizagem
}

\author{
Michel José Anzanello \\ Flávio Sanson Fogliatto \\ UFRGS
}

\begin{abstract}
Resumo
Em ambientes de alta customização, uma grande variedade de modelos de produtos é demandada pelos consumidores. Essa condição exige uma rápida adequação dos meios produtivos às especificações do próximo modelo a ser produzido. Tal situação, contudo, pode acarretar perdas consideráveis em relação aos níveis de produção e qualidade, em decorrência da pouca habilidade dos trabalhadores nos ciclos iniciais de produção de um novo modelo. Assim, a modelagem do processo de aprendizado de trabalhadores atuando sobre cada modelo de produto pode auxiliar a gerência na alocação de modelos de produtos a equipes de trabalhadores, minimizando as perdas verificadas nos primeiros ciclos de produção. Este artigo apresenta uma metodologia baseada na utilização de curvas de aprendizagem como balizadoras da alocação de modelos de produtos a equipes de trabalhadores. Os diversos modelos de produtos são agrupados em famílias de acordo com suas características similares, permitindo uma redução na coleta de dados. A alocação das famílias às equipes é realizada através da análise das curvas de aprendizagem. Duas formas de alocação são apresentadas, de acordo com a duração da corrida de produção. A metodologia proposta é ilustrada através de um estudo de caso em uma indústria do setor calçadista.
\end{abstract}

\section{Palavras-chave}

Curvas de aprendizagem, customização, alocação de produtos a equipe, programação da produção, indústria de calçados.

\section{Assignment of product models to worker teams using learning curve models}

\begin{abstract}
In highly customized markets a large variety of product models are typically demanded by customers. That requires fast setup of production resources to comply with specifications of the next model to be produced. Such compliance, however, may cause considerable production and quality losses due to workers' poor performance during the initial production runs of a new model. Therefore, modeling workers' learning upon exposure to each product model may help production managers to define the best assignment scheme for models and workers, such that losses in the initial stages of production are minimized. This paper presents a methodology that uses learning curves to guide the best assignment of product models to teams of workers. Product models are first clustered into families based on their similarities, leading to a smaller data collection. Then allocation of product families to teams is carried based on the analysis of their corresponding learning curves. Two courses of action are then proposed, depending on whether the production batch will lead to longer or shorter production runs. The proposed methodology is illustrated in a case study from the shoe manufacturing industry.
\end{abstract}

\section{Key words}

Learning curves, customization, assignment of products to teams, production management, shoe manufacturing industry. 


\section{INTRODUĈ̣̃O}

O mercado tem imposto severas condições para a sobrevivência das empresas em um cenário altamente competitivo, demandando esforços contínuos na melhoria da qualidade, eficiência e flexibilidade dos sistemas produtivos (DE RON, 1998). A fim de buscar novas estratégias capazes de diferenciar seus produtos, as empresas passaram a investir em produção baseada em uma maior variedade de catálogo. A oferta de uma maior gama de opções de produto passou a ser encarada como uma característica desejada pelos clientes, que em muitos casos estão dispostos a pagar preços mais elevados por itens diferenciados (DA SILVEIRA et al., 2001).

da redução de custos de produção e previsão de desempenho dos trabalhadores quando submetidos a uma nova operação (TEPLITZ, 1991).

Entretanto, apesar da vasta aplicação das curvas de aprendizagem e do conhecimento consolidado a respeito da variabilidade humana na realização de tarefas manuais, há uma carência de métodos na literatura que permitam avaliar a adaptação dos trabalhadores às operações e possibilitem a alocação dos mesmos a diferentes modelos de produtos, cujas corridas de produção apresentam durações distintas.

Este artigo busca suprir tal lacuna, propondo a utilização de curvas de aprendizagem para minimizar a variabilidade humana na realização de operações relativas a novos produtos. As curvas de aprendizagem são utilizadas como balizadoras da alocação de produtos a equipes de trabalhadores, permitindo o direcionamento dos diversos modelos de produtos às equipes mais aptas à realização de suas operações. Os parâmetros da curva de aprendizagem permitem analisar quantitativamente a adaptação dos trabalhadores às operações avaliadas, possibilitando a identificação de perfis distintos

A produção customizada resulta em uma grande variedade de itens e alteração constante no tamanho dos lotes de produtos demandados, exigindo flexibilidade dos recursos e interação dos meios produtivos (maquinário e mão-de-obra) para se adaptarem de maneira rápida e precisa às modificações impostas (DA SILVEIRA et al., 2001). A alteração freqüente nos modelos dos produtos submete o trabalhador a novas tecnologias e habilidades, desencadeando um processo de aprendizado a cada nova operação que lhe é imposta (TOWILL, 1990).

A adaptação do trabalhador a um novo produto ou serviço, contudo, pode impor consideráveis perdas ao sistema produtivo (exemplificadas pelos baixos índices de produtividade e qualidade obtidos nos primeiros ciclos de um novo produto), tornando útil a avaliação do processo de aprendizado. Nesse contexto, a curva de aprendizagem tem se mostrado uma ferramenta útil no monitoramento do desempenho do trabalhador submetido a uma nova operação, avaliando seu progresso à medida que repetições são efetuadas. Curvas de aprendizagem foram propostas inicialmente por Wright em 1922, através da verificação da redução do tempo e custo necessários para a montagem de aviões durante a Primeira Guerra Mundial. Desde então, as curvas de aprendizagem têm sido utilizadas para avaliação do tempo demandado para conclusão de corridas de produção, estimação de aprendizado que poderão ser administrados de acordo com os modelos de produtos demandados.

$\mathrm{O}$ artigo traz três contribuições originais, relevantes à área de Planejamento e Controle da Produção: (i) uma metodologia que sistematiza a utilização de curvas de aprendizagem para promover um escalonamento otimizado da produção em linhas multimodelos; (ii) a utilização de análise de regressão sobre parâmetros de curvas de aprendizagem, viabilizando a alocação de produtos com corridas curtas de produção a equipes de trabalhadores; e (iii) a otimização da coleta de dados para construção de curvas de aprendizagem agrupando modelos de produtos em clusters, conforme suas similaridades.

A metodologia proposta é ilustrada em uma aplicação no setor de costura de uma indústria calçadista, com o propósito de mensurar e avaliar o desempenho dos trabalhadores na fabricação de determinados modelos de calçados. Os diversos modelos são agrupados em famílias através de procedimentos de clusterização, permitindo a redução da quantidade de dados a serem coletados. As curvas são usadas para designar trabalhadores - ou equipes de trabalhadores - aptos a atuarem em determinadas famílias de modelos, de maneira a reduzir as perdas decorrentes das trocas de modelo de produto.

A estrutura deste artigo é a seguinte: apresentação de uma fundamentação teórica sobre modelos de curvas de 
aprendizagem, além de revisão de trabalhos desenvolvidos com propósitos similares aos deste artigo. Em seguida descreve-se a metodologia proposta e apresenta-se a aplicação da metodologia ao estudo de caso. Finalmente, a conclusão encerra o artigo.

\section{REFERENCIAL TEÓRICO}

Curvas de aprendizagem são representações matemáticas do desempenho de um trabalhador quando submetido a uma tarefa repetitiva. À medida que repetições são efetuadas, o trabalhador demanda menos tempo para execução da tarefa, seja pela familiaridade adquirida nos meios de produção, pela adaptação às ferramentas utilizadas ou pela descoberta de atalhos para realização da mesma (WRIGHT, 1936; TEPLITZ, 1991). Dentre as curvas de aprendizagem mais difundidas, merecem destaque os seguintes modelos:

- Modelos potenciais (Wright, Plateau, Stanford-B, DeJong e Curva S, entre outros);

- Modelos hiperbólicos;

- Modelos exponenciais.

O modelo de Wright constitui-se na curva de aprendizagem mais difundida, dada sua estrutura simples e boa capacidade de aderência a dados empíricos. A curva é representada por $y=C_{I} x^{b}$, onde $y$ indica o tempo (custo) acumulativo médio demandado, $C_{1}$ é o tempo (custo) da primeira unidade produzida, $x$ quantifica as unidades produzidas e $b$ é a declividade da curva, tal que $-1 \leq b \leq 0$.

O modelo de Plateau é composto pelo acréscimo de uma constante aditiva ao modelo de Wright $\left(y=C+C_{I} x^{b}\right)$, fazendo com que $y$ tenda a $C$ quando o volume de produção for elevado. $O$ valor da constante $C$ reproduz o desempenho do trabalhador ao atingir o estado estacionário, situação em que o processo de aprendizado está próximo da conclusão (YELLE, 1979; TEPLITZ, 1991).

A curva de aprendizagem proposta por Stanford-B é representada por $y=C_{1}(x+B)^{b}$, onde $B$ visa incorporar a experiência prévia do trabalhador na operação ao modelo de Wright (TEPLITZ, 1991; NEMBHARD; UZUMERI, 2000).

O modelo de DeJong analisa a influência da participação de maquinário no processo de aprendizado. A equação é representada por $y=C_{1}\left[M+(1-M) x^{b}\right]$, onde $M$ $(0 \leq \mathrm{M} \leq 1)$ é o fator de incompressibilidade e representa a proporção do tempo total de operação constituído por procedimentos automatizados (YELLE, 1979; BADIRU, 1992).

A curva $S$ contempla operações que necessitam da intervenção de máquinas e cuja análise do desempenho nas primeiras unidades assume importância para descrição do processo. A equação foi desenvolvida através da união dos modelos de DeJong e Stanford-B e é representada por $y=C_{l}\left[M+(1-M)(x+B)^{b}\right]$, onde os parâmetros assumem o mesmo significado dos modelos em que foi baseada (BADIRU, 1992; NEMBHARD; UZUMERI, 2000).

Curvas de aprendizagem de natureza hiperbólica e exponencial são constituídas por parâmetros diferenciados quando comparados aos modelos potenciais. Tais parâmetros permitem extrair maior quantidade de informação a respeito do processo de aprendizado do trabalhador, gerando previsões de produção mais precisas que as fornecidas pelas curvas potenciais.

Thurstone apud Mazur e Hastie (1978) e Kientzle apud Mazur e Hastie (1978) propuseram uma curva de aprendizagem hiperbólica constituída por três parâmetros:

$$
y=k\left(\frac{x+p}{x+p+r}\right)
$$

tal que $p+r>0$, e onde $y$ é o desempenho do trabalhador na execução de determinada tarefa, expresso em unidades produzidas após $x$ unidades de tempo de operação ( $y$ $\geq 0$ ); $x$ denota o tempo de operação na tarefa, expresso em unidades de tempo $(x \geq 0) ; k$ é o patamar máximo de desempenho a ser atingido quando a aquisição de conhecimento for integral, expresso em número de unidades produzidas por tempo de operação $(k \geq 0) ; p$ designa a experiência prévia do trabalhador na execução da tarefa em questão, expressa em unidades de tempo $(p \geq 0)$; e $r$ é o tempo de operação necessário para atingir $k / 2$, que corresponde à metade do patamar de desempenho máximo $(k)$. O parâmetro $r$ é expresso em unidades de tempo e indica a taxa de aprendizado do trabalhador. Valores elevados de $r$ indicam a necessidade de elevado tempo de operação para atingir determinado valor de $k$, sinalizando um processo de aprendizado lento (UZUMERI; NEMBHARD, 1998).

O parâmetro $r$ atua como parâmetro de forma da equação hiperbólica, podendo descrever três padrões diferentes de aprendizado. Quando $r>0$, a curva cresce até um valor limite de desempenho $k$. A velocidade de crescimento da curva é mais elevada quando $r$ apresenta baixos valores, ou seja, são necessárias poucas repetições (ou menor tempo de operação) para que o patamar $k / 2$ seja alcançado. Situações onde $r>0$ são típicas de trabalhadores com reduzida experiência prévia submetidos a novas operações. Quando $r \rightarrow 0$, a curva comporta-se como uma linha horizontal, descrevendo um processo onde não há aprendizado. Quando $r<0$, tem-se situações onde o desempenho, representado por uma curva decrescente, diminui com o transcorrer do processo. Esse caso 
indica a ocorrência de queda de desempenho, seja por fadiga ou por esquecimento quanto à tarefa (UZUMERI; NEMBHARD, 1998). A Figura 1 apresenta os perfis gerados pelo modelo hiperbólico de 3 parâmetros, de acordo com o módulo e magnitude do parâmetro $r$.

Uzumeri e Nembhard (1998) utilizaram o modelo hiperbólico para o monitoramento dos diversos perfis de aprendizado apresentados por uma população de trabalhadores submetidos a novas operações, através da análise dos parâmetros gerados pelas modelagens. Os autores concluíram que trabalhadores que apresentam rápida adequação à nova operação (baixo valor de $r$ ) tendem a atingir patamares de desempenho final $k$ inferiores aos dos trabalhadores que assimilam as operações de forma mais lenta (alto valor de $r$ ). De tal forma, é possível alocar trabalhadores com reduzidos valores de $r$ a operações de curta duração, enquanto que trabalhadores que apresentam elevados valores de $r$ devem ser direcionados a tarefas de mais longa duração, dados seus patamares elevados de desempenho final.

Dentre os modelos baseados em curvas de natureza exponencial, merecem destaque os modelos exponencial de 3 parâmetros e o de tempo constante. O modelo exponencial de 3 parâmetros é apresentado na equação (2).

$$
y=k\left(1-e^{-(x+p) / r}\right)
$$

Estudos realizados por Mazur e Hastie (1978) mostram que a equação (2) resulta em modelagens deficien- tes quando o trabalhador analisado é submetido a tarefas complexas e que demandam grande quantidade de novos conhecimentos. Em contrapartida, gera bons resultados em situações em que o trabalhador apresenta experiência prévia.

A similaridade na interpretação dos parâmetros $k, r$ e $p$ das equações (1) e (2), apesar das estruturas matemáticas distintas, levou Mazur e Hastie (1978) a desenvolverem uma série de experimentos buscando comparar os resultados previstos pelos modelos exponencial e hiperbólico. Os parâmetros $r$ e $p$ gerados pelas equações (1) e (2) apresentaram grandezas similares. As maiores diferenças foram verificadas no valor de desempenho final $(k)$ estipulado pelas equações. De maneira geral, o modelo exponencial subestima o parâmetro $k$, levando a previsões de desempenho inferiores aos valores verificados em aplicações práticas. O modelo hiperbólico forneceu valores de $k$ próximos aos verificados nos experimentos, além de oferecer melhores ajustes aos dados experimentais (maior coeficiente de determinação $R^{2}$ ). Mazur e Hastie (1978) concluíram que o modelo hiperbólico de 3 parâmetros mostrou-se eficiente para modelar qualquer seqüência de etapas de operações, sendo superior ao modelo exponencial em vários aspectos e possibilitando averiguar de maneira bastante precisa o processo de aprendizado do trabalhador.

O modelo de tempo constante foi desenvolvido por Towill (1990) e se baseia em uma estrutura semelhante à curva exponencial de 3 parâmetros, sendo apresentado na equação (3).

Figura 1: Perfis gerados pelo modelo hiperbólico de 3 parâmetros. (Adaptado de UZUMERI; NEMBHARD, 1998)

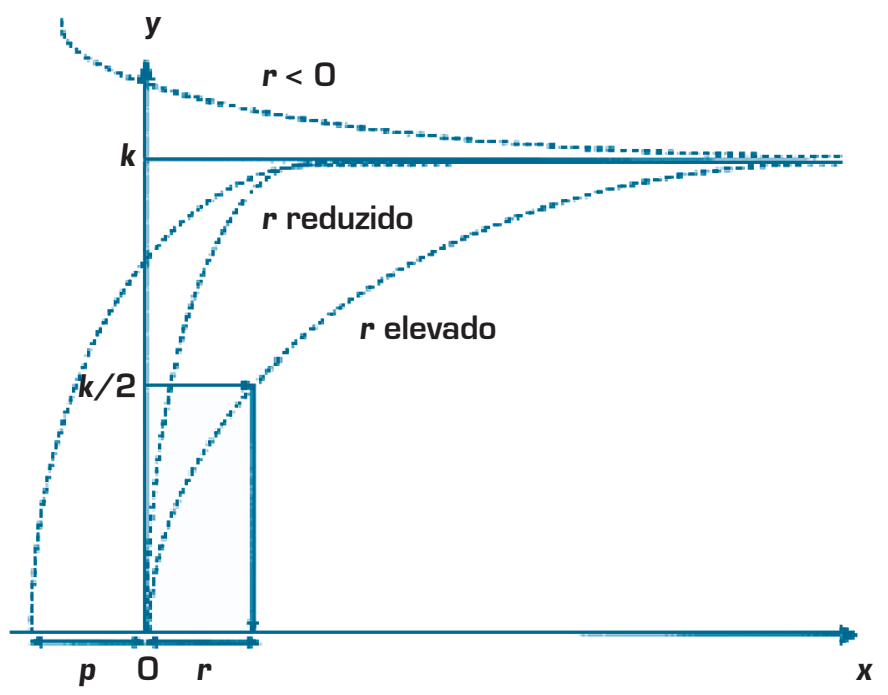




$$
y=y_{c}+y_{f}\left(1-e^{-t / \tau}\right)
$$

O modelo utiliza o tempo acumulativo de operação $(t)$ como variável independente, sendo seu significado idêntico ao número de unidades produzidas $(x)$ adotado pelos demais modelos de curvas. Essa adaptação possibilita a determinação simplificada do tempo demandado para a obtenção de determinado patamar de desempenho. Naim e Towill (1990) avaliaram o Modelo de Tempo Constante como uma ferramenta apropriada para descrição do aprimoramento de trabalhadores, dada sua aplicabilidade em tarefas de naturezas diversas. $\mathrm{O}$ modelo pode ser combinado com funções trigonométricas, sendo útil em situações onde há alternância entre tendência contínua de aprimoramento e variações cíclicas de desempenho decorrentes de fatores conhecidos. Segundo Towill (1990), o modelo é recomendado para situações em que a coleta de dados de desempenho se inicia depois de um pequeno período de adaptação do trabalhador à tarefa.

\section{METODOLOGIA PROPOSTA}

A metodologia proposta visa avaliar a adequação de equipes de trabalhadores às operações demandadas por produtos distintos, com o propósito de direcioná-los às equipes mais habilitadas à sua execução. A alocação é baseada na análise dos parâmetros gerados pelas curvas de aprendizagem. A Figura 2 apresenta as etapas metodológicas propostas.

A escolha dos trabalhadores (ou equipes) visa selecionar equipes para a coleta de dados de desempenho, as quais serão avaliadas na execução das operações demandadas pelos modelos de produtos analisados. Uma equipe pode ser composta por trabalhadores atuando na mesma operação ou executando operações distintas, como acontece em linhas de produção. O monitoramento de equipes com o propósito de alocação de produtos demanda que as equipes estejam previamente formadas e aptas à execução das operações demandadas na fabricação dos produtos analisados. As equipes não devem apresentar elevado percentual de rotatividade externa, o que poderia modificar o perfil de desempenho da equipe durante a análise. As equipes avaliadas serão identificadas pelo índice $i=1, \ldots, I$.

A seleção dos produtos a serem direcionados às equipes de trabalhadores para coleta de dados de desempenho deve priorizar os itens considerados mais representativos e rentáveis, de maneira a justificar a sua modelagem pelas curvas de aprendizagem. Cenários de produção

Figura 2: Fluxograma da metodologia proposta.

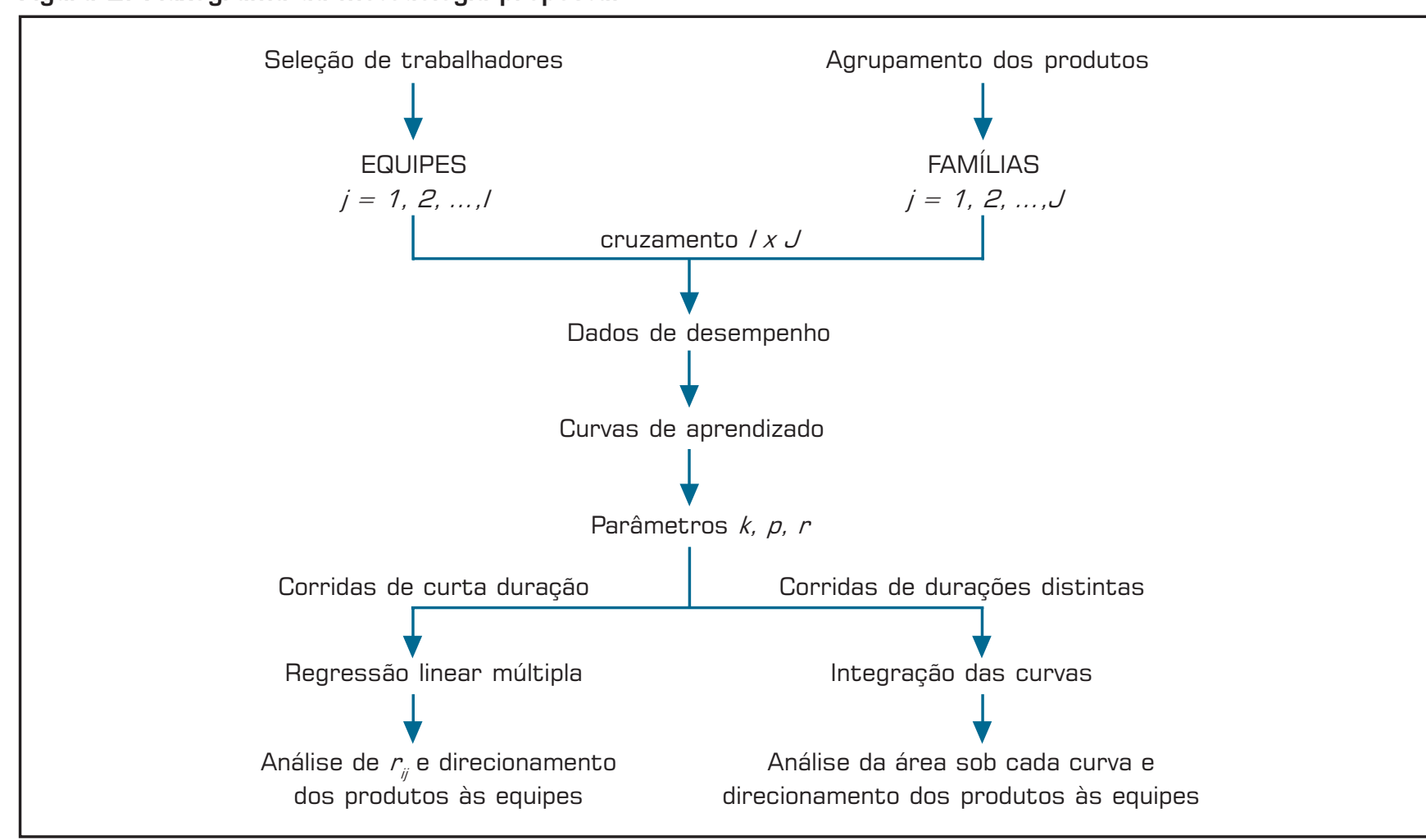


caracterizados por grande variedade de modelos de produtos possibilitam a formação de famílias de produtos com características similares, reduzindo a quantidade de dados a serem coletados.

As características selecionadas para a formação das famílias de produtos são definidas como variáveis de clusterização e devem ser relevantes para a formação de famílias representativas. Essas características estão relacionadas com particularidades físicas do produto, complexidade das operações envolvidas em sua fabricação e outras propriedades que permitam efetuar um agrupamento dos modelos. O procedimento de formação dos clusters (famílias de produtos) pode ser realizado através de software estatístico. A análise de clusters confere significância estatística às famílias, tal que as conclusões obtidas para um produto pertencente a uma família possam ser estendidas para os demais integrantes daquela família (JOBSON, 1992; HAIR et al., 1995). As famílias formadas serão identificadas pelo índice $j=1, \ldots, J$.

modelo mais adequado para o monitoramento do processo de aprendizado. Os modelos hiperbólico de 3 parâmetros e exponencial de 3 parâmetros [equações (1) e (2), respectivamente] foram escolhidos tomando-se por base a classificação de Nembhard e Uzumeri (2000), segundo a qual essas equações apresentam os melhores resultados de eficiência e estabilidade frente aos demais modelos analisados em vários cenários testados. Os parâmetros que compõem as duas equações $(k, p$ e $r)$ apresentam significados idênticos, possibilitando comparações diretas entre os resultados obtidos pelas modelagens. A terceira equação testada é o modelo de tempo constante [equação (3)], sendo sua estrutura matemática similar ao modelo exponencial. Os parâmetros constituintes da equação de tempo constante $\left(\mathrm{Y}_{\mathrm{c}}, \mathrm{Y}_{\mathrm{f}}\right.$ e $\left.\tau\right)$ possuem definições distintas dos parâmetros dos modelos hiperbólico e exponencial. Mesmo assim, há analogia entre o significado prático dos parâmetros, de forma a possibilitar comparações entre os resultados das modelagens oriundas das três equações.

Os parâmetros das três equações testaA adaptação do trabalhador a um novo perdas ao sistema produtivo, tornando útil a avaliação do processo de aprendizado.

As $J$ famílias de produtos formadas são cruzadas com as $I$ equipes de trabalhadores selecionadas como amostra. A idéia-chave é obter dados de desempenho de cada equipe na execução de operações "gargalo" de aprendizado (operações de maior complexidade percebida) relativas a cada família de produtos. A coleta dos dados de desempenho da operação "gargalo" deve ter início nos primeiros ciclos de fabricação do produto, sendo executada até que a produtividade horária atinja o estado estacionário (dados de desempenho com baixa variabilidade), sinalizando que o processo de aprendizado do trabalhador está próximo da conclusão. A coleta é operacionalizada através da contagem do número de unidades produzidas em cada intervalo de tempo de operação, podendo ser realizada através de contadores eletrônicos ou com o auxílio de planilha e cronômetro. Produtos em processamento no momento do fechamento de um intervalo são automaticamente computados no intervalo seguinte.

Os dados de desempenho coletados do processo são modelados por três modelos de curvas, sendo a variável de resposta expressa em número de unidades produzidas por tempo de operação. Objetiva-se, de tal forma, avaliar a aderência de cada modelo aos dados, selecionando o rente à i-ésima equipe de trabalhadores. das são obtidos através de regressão nãolinear por intermédio de software de análise estatística. Os dados de desempenho são modelados como variável dependente $(y)$ do tempo acumulado de atuação do trabalhador na operação analisada $(x)$. Assim, uma família de produtos $j$ gera um conjunto de parâmetros $k_{i j}$, $p_{i j}$ e $r_{i j}$, refe-

Os critérios utilizados para avaliar a aderência dos modelos de curvas de aprendizagem aos dados de desempenho são: (i) soma dos quadrados dos erros (SQE), sendo os erros obtidos pela diferença entre os valores reais e os preditos pela curva; ( ii) coeficiente de determinação $\left(R^{2}\right)$; e (iii) capacidade preditiva das curvas em uma amostra de validação, mensurada através do percentual de desvio entre o valor predito pelas curvas para um instante de operação em estado estacionário e o valor real obtido para a amostra de validação. A análise dos três critérios de aderência permite selecionar o modelo de curva de aprendizagem mais adequado para descrição do desempenho das equipes de trabalhadores nas operações analisadas. As etapas seguintes da metodologia referem-se aos procedimentos e análises a serem realizados sobre os parâmetros do modelo de curva de aprendizagem selecionado.

Os parâmetros do modelo selecionado são analisados de duas maneiras, de acordo com a duração da corrida de produção: análise de regressão sobre o parâmetro $r$ de forma isolada, voltada para corridas de produção de curta duração, e análise das curvas construídas a partir dos 
parâmetros, aplicável em corridas de durações distintas. A estimativa da duração da corrida de produção pode ser feita através da programação de produção da empresa, dividindo-se o tamanho do lote a ser produzido pela capacidade de produção conhecida. Cabe aos especialistas classificarem as durações em curtas ou longas, de acordo com o ambiente de produção estudado.

\section{Alocação de modelos com corridas de curta duração}

Em casos de corridas de curta duração, a proposta é utilizar a análise de regressão sobre o parâmetro de taxa de aprendizado $(r)$ como balizadora da alocação de modelos a equipes de trabalhadores. A análise isolada do parâmetro $r$ é justificada pela busca de trabalhadores que se adaptem com maior rapidez às operações demandadas, independentemente do desempenho final apresentado por esses trabalhadores. Esta consideração é adequada em ambientes cujas corridas de produção são curtas e o número de unidades produzidas insuficiente para que trabalhadores com reduzida experiência atinjam o patamar de desempenho máximo na operação. Dessa maneira, propõe-se relacionar o parâmetro $r$ através de um modelo de regressão linear múltipla, composto pelas seguintes variáveis independentes: $(i)$ equipe de trabalhadores $\left(X_{1}\right)$, (ii) família de produtos $\left(X_{2}\right)$, e (iii) interação entre equipe de trabalhadores e família de produtos $\left(X_{1} X_{2}\right)$. A representação genérica do modelo é apresentada na equação (4).

$$
r_{i j}=b_{0}+b_{1} X_{1}+b_{2} X_{2}+b_{3} X_{1} X_{2}
$$

O parâmetro $r_{i j}$ é a variável dependente do modelo de regressão, onde $i$ representa a equipe e $j$ representa a família de produto considerada. As variáveis $X_{1}$ e $X_{2}$ são substituídas por escalas codificadas de números inteiros positivos referentes a cada equipe de trabalhadores e a cada família de produtos, respectivamente. Os valores de escala devem estar atrelados aos indicadores da equipe ou do produto em questão. Por exemplo, a codificação da equipe de trabalhadores pode refletir seu desempenho, conforme avaliado subjetivamente por especialistas; assim, a equipe cujo $X_{1}=1$ deve ter desempenho inferior ao da equipe com $X_{1}=2$. $O$ valor da variável $X_{1} X_{2}$ é obtido através da multiplicação das variáveis $X_{1}$ e $X_{2}$, representando a interação entre equipe e família de produtos.

A determinação das constantes $b_{0}, b_{1}, b_{2}$ e $b_{3}$ na equação (4) pode ser realizada através de software de análise estatística, o que permite avaliar a significância de cada variável independente e a magnitude da sua influência sobre a taxa de aprendizado do trabalhador. $\mathrm{O}$ menor valor de $r_{i j}$ gerado pela regressão refere-se à equipe que apresenta a melhor taxa de aprendizado para a família de produtos em questão, sendo a mais recomendada para a execução da operação. A elaboração de um modelo de regressão é útil quando se opera com um grande número de produtos e equipes, o que inviabilizaria a observação de todos os cruzamentos possíveis.

\section{Alocação de modelos com corridas de duração variada}

Em casos de corridas de duração variada, ou quando a caracterização da duração da corrida é incerta por parte dos especialistas, a proposta é integrar a curva de aprendizagem no intervalo de análise e utilizar a área resultante como balizadora da alocação de modelos a equipes de trabalhadores. As curvas de aprendizagem a serem analisadas são construídas a partir dos parâmetros $\bar{k}_{i j}, \bar{p}_{i j}$ e $\bar{r}_{i j}$, obtidos pela média dos parâmetros $k_{i j}, p_{i j}$ e $r_{i j}$, referentes aos cruzamentos repetidos de uma equipe i com uma família j de produtos (observe que na proposta para corridas de curta duração o cálculo dos parâmetros médios não é necessário, pois a modelagem de regressão faz uso direto das repetições). Assim, cada equipe possui um conjunto de parâmetros médios que permitem avaliar o processo de aprendizado e efetuar previsões de desempenho para horizontes de tempos variados. A utilização de parâmetros médios busca capturar os vários comportamentos de desempenho apresentados por uma equipe atuando em produtos de uma mesma família, incorporando eventuais variações de desempenho.

As curvas geradas para cada família são analisadas em um gráfico comparativo, sendo que a abscissa das curvas representa o tempo de operação na tarefa analisada e a ordenada indica o número de unidades produzidas por tempo de operação. A análise do aprendizado é feita através da comparação do nível de produção previsto pela curva de aprendizagem gerada para cada equipe atuando sobre produtos de uma dada família. A avaliação visual da curva, entretanto, não é suficiente para quantificar de forma precisa a produção alcançada pelas equipes com o avanço do processo. Assim, a avaliação é realizada pela comparação das áreas formadas sob as curvas de aprendizagem das equipes monitoradas. As áreas podem ser obtidas através da integração da equação da curva selecionada, podendo ser realizada de forma numérica ou analítica.

A análise do resultado da integração das curvas especifica, para um tempo $t$ de duração estimada para a corrida de produção, o valor da área gerada por cada equipe atuando em uma família de produtos. A equipe detentora da maior área apresenta o maior nível de produtividade, sendo a mais adequada para a execução dos produtos daquela família. 


\section{Comparativo dos procedimentos propostos}

A análise isolada do parâmetro $r$ é justificada em corridas curtas de produção, as quais não oferecem tempo de operação suficiente (ou número de repetições suficientes) para que trabalhadores com reduzida experiência atinjam o patamar de desempenho máximo na operação. Dessa forma, desaconselha-se a utilização do parâmetro $k$ nos procedimentos de alocação e priorizam-se as equipes detentoras do menor valor de $r$, as quais demandam menor quantidade de tempo para atingir determinado padrão de desempenho.

O método de análise das áreas, por sua vez, captura a influência dos parâmetros $k, p$ e $r$, podendo ser utilizado em corridas de produção com durações variadas. Corridas caracterizadas como longas utilizam o parâmetro $k$ como estimador do desempenho estacionário do trabalhador, uma vez que o tempo de duração de produção do modelo é suficiente para que esse estágio seja atingido. Valores elevados de $k$ determinam maiores áreas sob a curva, apontando a equipe detentora dos maiores níveis de produtividade como mais adequada para a execução dos modelos avaliados. A análise em corridas de curta duração é efetuada de forma semelhante, sendo que menores valores do parâmetro $r$ agem no sentido de aumentar a área sob a curva. A equipe detentora da maior área (maiores níveis de produtividade) é escolhida para realização dos modelos daquela família.

\section{ESTUDO DE CASO}

A metodologia proposta é ilustrada no setor de costura de uma empresa do setor calçadista. Dado o elevado número de calçados analisados (20 modelos), utilizou-se a análise de clusters para formar três famílias de modelos (Fácil, Média e Difícil), de acordo com as características dos procedimentos de costura demandados pelos modelos. As características foram avaliadas subjetivamente por especialistas utilizando uma escala de 1 a 3 , sendo que 3 representa alta complexidade do calçado na característica analisada. A Tabela 1 apresenta os valores atribuídos aos calçados e a família em que cada modelo foi inserido.

Selecionaram-se três equipes de trabalhadores (Equipe 1, Equipe 2 e Equipe 3), para as quais foram direcionados os calçados pertencentes às três famílias formadas. Os dados de desempenho, coletados na forma de número de calçados produzidos por intervalo de 10 minutos de operação, foram modelados pelas equações (1), (2) e (3) através do software SPSS v.8.0. Os parâmetros gerados pelas curvas analisadas, bem

Tabela 1: Atributos das características avaliadas e família em que cada modelo foi inserido.

\begin{tabular}{|c|c|c|c|c|c|c|c|c|}
\hline \multirow{2}{*}{ GALÇADO } & \multicolumn{7}{|c|}{ CARACTERÍSTICAS } & \multirow{2}{*}{ FAMÍLIA } \\
\hline & COMPLEXIDADE & COSTURA & ENFEITE & FORRO & MATERIAL & $N^{\circ}$ PEÇAS & SAP/OUTRO & \\
\hline Calçado 1 & 1 & 1 & 1 & 1 & 1 & 2 & 2 & Fácil \\
\hline Calçado 2 & 2 & 1 & 1 & 2 & 2 & 1 & 1 & Fácil \\
\hline Calçado 3 & 1 & 1 & 1 & 2 & 1 & 1 & 2 & Fácil \\
\hline Calçado 4 & 2 & 2 & 1 & 1 & 1 & 2 & 1 & Fácil \\
\hline Calçado 5 & 1 & 1 & 2 & 2 & 1 & 2 & 1 & Fácil \\
\hline Calçado 6 & 1 & 1 & 1 & 1 & 1 & 1 & 1 & Fácil \\
\hline Calçado 7 & 2 & 1 & 1 & 1 & 2 & 1 & 1 & Fácil \\
\hline Calçado 8 & 2 & 2 & 3 & 1 & 1 & 1 & 1 & Média \\
\hline Calçado 9 & 2 & 2 & 2 & 1 & 2 & 2 & 1 & Média \\
\hline Calçado 10 & 2 & 2 & 2 & 1 & 2 & 1 & 1 & Média \\
\hline Calçado 11 & 2 & 1 & 3 & 1 & 1 & 2 & 1 & Média \\
\hline Calçado 12 & 2 & 2 & 2 & 1 & 2 & 2 & 1 & Média \\
\hline Calçado 13 & 2 & 1 & 2 & 1 & 2 & 2 & 1 & Média \\
\hline Calçado 14 & 2 & 1 & 2 & 1 & 2 & 2 & 1 & Média \\
\hline Calçado 15 & 3 & 2 & 3 & 2 & 3 & 2 & 1 & Difícil \\
\hline Calçado 16 & 3 & 1 & 3 & 2 & 3 & 2 & 1 & Difícil \\
\hline Calçado 17 & 2 & 2 & 2 & 2 & 2 & 3 & 1 & Difícil \\
\hline Calçado 18 & 2 & 2 & 3 & 2 & 3 & 2 & 1 & Difícil \\
\hline Calçado 19 & 2 & 2 & 3 & 2 & 2 & 2 & 2 & Difícil \\
\hline Calçado 20 & 3 & 2 & 3 & 2 & 2 & 3 & 1 & Difícil \\
\hline
\end{tabular}


como as equipes nas quais cada calçado foi elaborado, são apresentados na Tabela 2.

Os critérios de aderência, apresentados na Tabela 3, indicam que o modelo hiperbólico apresentou resultados de desempenho superiores aos modelos exponencial e de tempo constante nas modelagens realizadas. $\mathrm{O}$ modelo hiperbólico resultou na menor média da soma dos quadrados dos erros (SQE) e na maior capacidade preditiva em uma amostra de validação (medidos em termos de desvio), quando comparado aos demais modelos avaliados. O coeficiente de determinação $\left(R^{2}\right)$ apresentou valores similares para os 3 modelos, inviabilizando sua utilização como critério de seleção do melhor modelo. A ausência de valores de desvio, como, por exemplo, nos Calçados 4 e 11, corresponde a modelos com corridas de produção curtas (duração inferior a dois dias), o que impossibilitou a coleta de dados para amostra de validação. De tal forma, os parâmetros do modelo hiperbólico são utilizados nas etapas seguintes da análise.

Os parâmetros gerados pela equação hiperbólica foram analisados através de dois métodos distintos: análise do parâmetro $r_{i j}$ (regressão linear múltipla) e análise das curvas associadas a cada família de modelos de calçado (mais especificamente, analisando as áreas geradas pelas curvas). A primeira forma é indicada para análises em corridas de produção de curta duração e a segunda pode ser utilizada em corridas de durações distintas.

\section{Análise de regressão sobre o parâmetro de taxa de aprendizado}

As variáveis independentes consideradas na análise de regressão representam as equipes e as famílias de calçados analisadas. A escala adotada para a identificação das 3 equipes baseou-se na média de idade dos componentes de cada equipe monitorada (dados fornecidos pela empresa), sendo que ao maior valor de média foi atribuído o maior valor de escala. A Tabela 4 apresenta os valores associados às equipes. As três famílias de calçados na Tabela 5 foram assim codificadas: Difícil $=3$, Média $=2$ e Fácil $=1$, sendo os valores atribuídos de acordo com a complexidade da família em questão.

As constantes $b_{0}, b_{1}, b_{2}$ e $b_{3}$ da equação (4) foram estimadas com o auxílio do software SPSS v.8.0, através da opção de regressão linear. O coeficiente de determina-

Tabela 2: Parâmetros gerados pelos três modelos de curva de aprendizagem avaliados.

\begin{tabular}{|c|c|c|c|c|c|c|c|c|c|c|c|}
\hline \multirow[t]{2}{*}{ FAMÍLIA } & \multirow[t]{2}{*}{ GALÇADO } & \multicolumn{3}{|c|}{$\begin{array}{l}\text { HIPERBÓLICO } 3 \\
\text { PARÂMETROS }\end{array}$} & \multicolumn{3}{|c|}{$\begin{array}{l}\text { EXPONENCIAL } 3 \\
\text { PARÂMETROS }\end{array}$} & \multicolumn{3}{|c|}{$\begin{array}{c}\text { TEMPO } \\
\text { CONSTANTE }\end{array}$} & \multirow[t]{2}{*}{ EQUIPE } \\
\hline & & $k$ & $p$ & $r$ & $k$ & $p$ & $r$ & $y_{c}$ & $y_{f}$ & $\tau$ & \\
\hline \multirow{7}{*}{ Fácil (1) } & Calçado 1 & 11,4 & 72,5 & 147 & 11,5 & 118 & 281 & 3,9 & 7,6 & 282 & 1 \\
\hline & Calçado 2 & 12,3 & 88 & 145 & 9,3 & 101 & 144 & 4,7 & 4,6 & 144 & 1 \\
\hline & Calçado 3 & 8,1 & 105 & 167 & 7,2 & 169 & 281 & 3,2 & 3,9 & 281 & 2 \\
\hline & Calçado 4 & 12,1 & 54,5 & 156 & 10,1 & 77,8 & 195 & 3,3 & 6,8 & 195 & 2 \\
\hline & Calçado 5 & 18,9 & 29 & 44 & 17 & 58,8 & 88,1 & 8,3 & 8,7 & 88,1 & 2 \\
\hline & Calçado 6 & 18,7 & 8,2 & 10,5 & 16,8 & 17,8 & 22,7 & \multicolumn{3}{|c|}{ não convergiu } & 3 \\
\hline & Calçado 7 & 6,4 & 94,8 & 123 & 5,1 & 115 & 142 & 2,8 & 2,3 & 142 & 3 \\
\hline \multirow{7}{*}{ Médio (२) } & Calçado 8 & 14,7 & 13 & 71,8 & 10,5 & 19,9 & 60,6 & 2,3 & 8,2 & 60,6 & 1 \\
\hline & Calçado 9 & 15,1 & 5,1 & 42,6 & 11,5 & 7,6 & 41,6 & 1,9 & 9,5 & 41,6 & 1 \\
\hline & Calçado 10 & 18,7 & 29,5 & 26,3 & 16,4 & 49,3 & 51 & 10,2 & 6,2 & 51 & 1 \\
\hline & Calçado 11 & 7,1 & 20,4 & 59,6 & 6,7 & 58,2 & 135 & 2,4 & 4,4 & 135 & 2 \\
\hline & Calçado 12 & 19,7 & 8,4 & 80 & 15,3 & 13,9 & 82,9 & 2,4 & 13 & 82,9 & 2 \\
\hline & Calçado 13 & 43 & 24,6 & 30,9 & 37,3 & 40,6 & 53 & 19,9 & 17,3 & 53,1 & 3 \\
\hline & Calçado 14 & 10,2 & 7,5 & 45,1 & 8,5 & 17,2 & 61,2 & 2,1 & 6,4 & 61,2 & 3 \\
\hline \multirow{6}{*}{ Difícil (3) } & Calçado 15 & 9,8 & 77,9 & 68,7 & 9,1 & 161 & 180 & 5,4 & 3,7 & 180 & 1 \\
\hline & Calçado 16 & 4,5 & 19,2 & 64,9 & 4 & 50,2 & 122 & 1,3 & 2,6 & 122 & 2 \\
\hline & Calçado 17 & 17,6 & 22,9 & 36,8 & 14,8 & 36,1 & 55,1 & 7,1 & 7,7 & 55,1 & 2 \\
\hline & Calçado 18 & 11,1 & 37,1 & 221 & 7,3 & 38,5 & 158 & 1,6 & 5,8 & 158 & 3 \\
\hline & Calçado 19 & 27,1 & 29,3 & 19,3 & 24,4 & 53,2 & 46,2 & 16,7 & 7,7 & 46,3 & 3 \\
\hline & Calçado 20 & 8,8 & 36 & 52 & 7,8 & 75,9 & 105 & 4 & 3,8 & 105 & 3 \\
\hline
\end{tabular}


ção $\left(R^{2}\right)$ obtido foi de 0,21 , indicando uma baixa aderência do modelo de regressão aos dados analisados. O modelo gerado é apresentado na equação (5).

$$
r_{i j}=266,5-70,2 X_{1}-93,5 X_{2}+34,3 X_{1} X_{2}
$$

A análise da grandeza das constantes mostra que alterações na variável $X_{2}$ acarretam as maiores diminuições no valor de $r_{i j}$, revelando que as maiores variações na taxa de aprendizado do trabalhador são decorrentes da modificação da família em operação. A variável $X_{1}$ opera no mesmo sentido, porém sua magnitude é menor do que
$X_{2}$. A variável de interação $\left(X_{1} X_{2}\right)$ atua de forma a aumentar o valor da taxa de aprendizado.

Os valores de $r_{i j}$ gerados pelo modelo de regressão para as equipes e famílias analisadas são apresentados na Tabela 5. Os desvios relativos entre os valores modelados pela curva de aprendizagem e os valores gerados pelo modelo de regressão são calculados utilizando-se o parâmetro $r_{i j}$ modelado médio, obtido através da média dos parâmetros $r_{i j}$ para cruzamentos repetidos de uma equipe $i$ com uma família $j$ de produtos.

A significância das variáveis independentes é apresentada na Tabela 6. Considerando um grau de confiança

Tabela 3: Resumo dos erros (SQE), coeficientes de determinação $\left(\boldsymbol{R}^{2}\right)$ e desvios resultantes das modelagens.

\begin{tabular}{|c|c|c|c|c|c|c|c|c|c|c|}
\hline \multirow[t]{2}{*}{ FAMÍLIA } & \multirow[t]{2}{*}{ CALÇADO } & \multicolumn{3}{|c|}{$\begin{array}{l}\text { HIPERBÓLICO } 3 \\
\text { PARÂMETROS }\end{array}$} & \multicolumn{3}{|c|}{$\begin{array}{l}\text { EXPONENCIAL } 3 \\
\text { PARÂMETROS }\end{array}$} & \multicolumn{3}{|c|}{$\begin{array}{c}\text { TEMPO } \\
\text { CONSTANTE }\end{array}$} \\
\hline & & SQE & $R^{2}$ & DESVIO & SQE & $\boldsymbol{R}^{2}$ & DESVIO & SQE & $R^{2}$ & DESVIO \\
\hline \multirow{7}{*}{ Fácil (1) } & Calçado 1 & 4,4 & 0,6 & $8 \%$ & 4,4 & 0,6 & $19 \%$ & 4,4 & 0,6 & $19 \%$ \\
\hline & Calçado 2 & 2,77 & 0,76 & $5 \%$ & 2,75 & 0,76 & $-16 \%$ & 2,75 & 0,76 & $-16 \%$ \\
\hline & Calçado 3 & 4,9 & 0,64 & $-5 \%$ & 4,9 & 0,64 & $-13 \%$ & 4,9 & 0,64 & $-12 \%$ \\
\hline & Calçado 4 & 8,1 & 0,69 & - & 8,1 & 0,69 & - & 8,1 & 0,69 & - \\
\hline & Calçado 5 & 10,7 & 0,77 & $3 \%$ & 10,9 & 0,77 & $-6 \%$ & 10,9 & 0,77 & $-6 \%$ \\
\hline & Calçado 6 & 2,53 & 0,87 & $3 \%$ & 2,38 & 0,87 & $-7 \%$ & \multicolumn{3}{|c|}{ não convergiu } \\
\hline & Calçado 7 & 2,47 & 0,49 & $22 \%$ & 2,47 & 0,5 & $2 \%$ & 2,47 & 0,5 & $2 \%$ \\
\hline \multirow{7}{*}{ Médio (२) } & Calçado 8 & 6,4 & 0,82 & $22 \%$ & 6,3 & 0,82 & $-5 \%$ & 6,3 & 0,82 & $-5 \%$ \\
\hline & Calçado 9 & 4 & 0,91 & $23 \%$ & 3,8 & 0,91 & $-4 \%$ & 3,8 & 0,91 & $-5 \%$ \\
\hline & Calçado 10 & 2,8 & 0,87 & $-6 \%$ & 2,8 & 0,87 & $-16 \%$ & 2,8 & 0,87 & $-16 \%$ \\
\hline & Calçado 11 & 8,18 & 0,63 & - & 8,35 & 0,63 & - & 8,35 & 0,63 & - \\
\hline & Calçado 12 & 5,3 & 0,9 & $11 \%$ & 5,4 & 0,9 & $-4 \%$ & 5,4 & 0,9 & $-4 \%$ \\
\hline & Calçado 13 & 20,7 & 0,72 & - & 20,8 & 0,72 & - & 20,8 & 0,72 & - \\
\hline & Calçado 14 & 5,9 & 0,85 & $-3 \%$ & 6,3 & 0,84 & $-17 \%$ & 6,3 & 0,84 & $-17 \%$ \\
\hline \multirow{7}{*}{ Difícil (3) } & Calçado 15 & 5,0 & 0,63 & $-9 \%$ & 5,1 & 0,63 & $-12 \%$ & 5,1 & 0,63 & $-12 \%$ \\
\hline & Calçado 16 & 3,3 & 0,7 & $-18 \%$ & 3,5 & 0,69 & $-24 \%$ & 3,5 & 0,69 & $-26 \%$ \\
\hline & Calçado 17 & 5,5 & 0,8 & - & 5,5 & 0,8 & - & 5,5 & 0,8 & - \\
\hline & Calçado 18 & 4,38 & 0,84 & $-5 \%$ & 4,32 & 0,84 & $-30 \%$ & 4,32 & 0,84 & $-30 \%$ \\
\hline & Calçado 19 & 0,58 & 0,93 & - & 0,6 & 0,93 & - & 0,6 & 0,93 & - \\
\hline & Calçado 20 & 4,45 & 0,74 & - & 5,49 & 0,76 & - & 5,49 & 0,76 & - \\
\hline & MÉDIA & 5,62 & 0,76 & $10,7 \%$ & 5,71 & 0,76 & $12,1 \%$ & 5,89 & 0,75 & $12,7 \%$ \\
\hline
\end{tabular}

Tabela 4: Valores de escala atribuídos às equipes monitoradas.

\begin{tabular}{|c|c|c|}
\hline EQUIPE & IDADE MÉDIA & $\boldsymbol{x}_{\mathbf{1}}$ \\
\hline Equipe 1 & 35 & 1 \\
\hline Equipe 2 & 37 & 2 \\
\hline Equipe 3 & 40 & 3 \\
\hline
\end{tabular}


de $10 \%$, apenas $X_{2}$ é significativa, apesar de as significâncias de $X_{1}$ e $X_{1} X_{2}$ apresentarem valores bastante próximos ao limite de corte $(0,10)$. Esse resultado demonstra que a família de calçados $\left(X_{2}\right)$ é responsável pelas maiores variações sobre o parâmetro de taxa de aprendizado. A alteração de equipe $\left(X_{1}\right)$ impacta em menores variações de $r_{i j}$, o que pode ser justificado pelos padrões de desempenho semelhantes das equipes analisadas. A interação, a exemplo da equipe, também não é responsável por variações acentuadas em $r_{i j}$.

A análise dos valores de $r_{i j}$ gerados pelo modelo de regressão permite apontar a equipe mais habilitada à execução das operações relativas a cada família de produtos. Os valores mais baixos de $r_{i j}$ devem ser priorizados, visto que indicam a necessidade de menor tempo de operação para a obtenção de determinado patamar de desempenho. Por exemplo, a Equipe 3 é a mais apta à execução das operações pertencentes à família 1 (Fácil), visto que o seu parâmetro de taxa de aprendizado $\left(r_{31}=65,2\right)$ é inferior aos parâmetros apresentados pelas Equipes 1 e $2\left(r_{11}=137\right.$ e $r_{21}=101$, respectivamente).

Tabela 5: Escalas codificadas das famílias e equipes; e comparação entre os valores de $\boldsymbol{r}_{i j}$ modelados pela curva de aprendizagem e estimados pelo modelo de regressão.

\begin{tabular}{|c|c|c|c|c|c|c|}
\hline CALÇADO & $\begin{array}{c}\text { EQUIPE } \\
\left(X_{2}\right)\end{array}$ & $\begin{array}{l}\text { FAMÍLIA } \\
\left.\text { ( } X_{1}\right)\end{array}$ & $\begin{array}{c}r_{i j} \\
\text { MODELADO }\end{array}$ & $\begin{array}{c}\boldsymbol{r}_{i j} \text { MODELADO } \\
\text { MÉDIO }\end{array}$ & $\begin{array}{l}r_{i j} \text { ESTIMADO } \\
\text { (REGRESSÃO) }\end{array}$ & $\begin{array}{c}\text { DESVIO } \\
\text { RELATIVO }\end{array}$ \\
\hline Calçado 1 & 1 & 1 & 147 & \multirow[b]{2}{*}{146} & \multirow[b]{2}{*}{137} & \multirow[b]{2}{*}{$-6 \%$} \\
\hline Calçado 2 & 1 & 1 & 145 & & & \\
\hline Calçado 3 & 1 & 2 & 167 & \multirow{3}{*}{122} & \multirow{3}{*}{101} & \multirow{3}{*}{$-17 \%$} \\
\hline Calçado 4 & 1 & 2 & 156 & & & \\
\hline Calçado 5 & 1 & 2 & 44 & & & \\
\hline Calçado 6 & 1 & 3 & 10,5 & \multirow{2}{*}{66,8} & \multirow{2}{*}{65,2} & \multirow{2}{*}{$-2 \%$} \\
\hline Calçado 7 & 1 & 3 & 123 & & & \\
\hline Calçado 8 & 2 & 1 & 71,8 & \multirow{3}{*}{46,9} & \multirow{3}{*}{77,8} & \multirow{3}{*}{$66 \%$} \\
\hline Calçado 9 & 2 & 1 & 42,6 & & & \\
\hline Calçado 10 & 2 & 1 & 26,3 & & & \\
\hline Calçado 11 & 2 & 2 & 59,6 & \multirow{2}{*}{69,8} & \multirow{2}{*}{76,2} & \multirow{2}{*}{$9 \%$} \\
\hline Calçado 12 & 2 & 2 & 80 & & & \\
\hline Calçado 13 & 2 & 3 & 30,9 & \multirow{2}{*}{38} & \multirow{2}{*}{74,6} & \multirow{2}{*}{$96 \%$} \\
\hline Calçado 14 & 2 & 3 & 45,1 & & & \\
\hline Calçado 15 & 3 & 1 & 68,7 & 68,7 & 18,6 & $-73 \%$ \\
\hline Calçado 16 & 3 & 2 & 64,9 & \multirow[b]{2}{*}{50,9} & \multirow{2}{*}{51,3} & \multirow[b]{2}{*}{$1 \%$} \\
\hline Calçado 17 & 3 & 2 & 36,8 & & & \\
\hline Calçado 18 & 3 & 3 & 220 & \multirow{3}{*}{97,1} & \multirow{3}{*}{84} & \multirow{3}{*}{$-14 \%$} \\
\hline Calçado 19 & 3 & 3 & 19,3 & & & \\
\hline Calçado 20 & 3 & 3 & 52 & & & \\
\hline & & & & \multicolumn{2}{|c|}{ Desvio médio absoluto } & $32 \%$ \\
\hline
\end{tabular}

Tabela 6: Significâncias das variáveis independentes do modelo de regressão obtido.

\begin{tabular}{|c|c|}
\hline VARIÁVEL & SIGNIFIGÂNCIA \\
\hline Constante & 0,014 \\
\hline$X_{1}$ & 0,129 \\
\hline$X_{1}$ & 0,068 \\
\hline$X_{1} X_{2}$ & 0,119 \\
\hline
\end{tabular}




\section{Análise das curvas de aprendizagem construídas a partir dos parâmetros}

O parâmetro $k_{\mathrm{ij}}$, apresentado na Tabela 2, foi dividido pela produção programada pela empresa (expressa em número de unidades programadas em 10 minutos de operação, conforme apresentado na Tabela 7), gerando uma escala relativa de produção $\left(k_{i j}^{r}\right)$. Essa conversão tem por objetivo eliminar diferenças significativas de escala nos tempos de execução das diversas operações analisadas. As repetições de cruzamentos (mesma equipe atuando na mesma família) demandaram a determinação de $\bar{k}_{i j}^{r}$. A Tabela 7 exemplifica a obtenção dos parâmetros $\bar{k}_{12}^{r}, \bar{p}_{12} \mathrm{e}$ $\bar{r}_{12}$ gerados a partir dos dados de três calçados pertencentes à família Média $(j=2)$ e elaborados pela Equipe $1(i=1)$.

Os parâmetros médios foram utilizados para a construção de curvas, as quais foram organizadas em gráficos de acordo com a família de produtos. De tal forma, foram construídos três gráficos relativos às famílias Fácil, Média e Difícil, sendo cada gráfico composto por três curvas representativas do desempenho de cada equipe analisada. Na Figura 3 é apresentado o gráfico obtido para os calçados da família Difícil. Os gráficos das famílias Fácil e Média são gerados de forma semelhante.

Para a família Difícil, a análise da Figura 3 aponta a Equipe 1 como a mais adequada para a execução das operações em corridas de curta duração. Para corridas de longa duração, a Equipe 3 prontifica-se como a mais apropriada. Uma análise mais precisa pode ser obtida pela comparação das áreas geradas pela integração das curvas de aprendizagem para o intervalo de análise. Um exemplo de integração é apresentado na Tabela 8 , sendo que a equipe grifada representa a equipe mais adequada para a realização das operações em corridas com aquela duração. A análise da mesma tabela indica que produtos cuja corrida de produção tem duração inferior a 600 minutos devem ser direcionados para a Equipe 1 , enquanto que produtos com corridas de duração estimada superior a esse valor devem ser alocados à Equipe 3. Corridas com duração próxima a 600 minutos podem ter seus produtos alocados às Equipes 1 ou 3, sem haver diferença significativa nos níveis de produção resultantes.
A análise das áreas para as demais famílias de calçados é realizada de forma semelhante, sendo que os calçados classificados como Médios devem ser direcionados à Equipe 1 e os calçados da família Fácil devem ser alocados à Equipe 3.

De tal forma, as alocações sugeridas pelos dois métodos (regressão e integração) mostraram-se coincidentes em dois dos três casos analisados, apontando a Equipe 3 como a mais habilitada para a execução das operações relativas à família Fácil e a Equipe 1 para corridas curtas da família Difícil. As curvas geradas pelas Equipes 1 e 3 na execução da família mais complexa apresentaram um cruzamento decorridos 600 minutos de operação, significando que a primeira equipe apresentou aprendizado mais acentuado nas primeiras horas de atividade, mas foi superada pela Equipe 3 com o transcorrer do processo. Por conta deste cruzamento, confirma-se a abrangência de avaliação de cada método segundo a duração da corrida de produção: a regressão aponta a Equipe 1 como a que apresenta melhor aprendizado na partida do processo, mas não prevê a superação desse aprendizado pela Equipe 3 em corridas mais longas (situação prevista pelo método da integração).

Não houve concordância de alocação em relação à família Média, para a qual os métodos indicaram equipes distintas. Tal discrepância pode ser justificada pela similaridade de comportamento das curvas apresentadas pelos calçados da família Média, sendo que essa similaridade não é captada pelo método de análise isolada de $r_{i j}$ devido à baixa aderência obtida pela regressão $\left(R^{2}=0,21\right)$. Nessa situação, o resultado gerado pelo método baseado na análise das áreas deve ser priorizado, fazendo com que os calçados inseridos na família Média sejam direcionados para a Equipe 1. O Quadro 1 apresenta o resumo das alocações das famílias às equipes sugeridas pelos dois métodos.

\section{CONCLUSÃO}

Este artigo teve como objetivo principal a apresentação de uma metodologia de utilização de curvas de aprendizagem como balizadoras da alocação de famílias de produtos

Tabela 7: Procedimento de obtenção dos parâmetros médios para 3 modelos de calçados.

\begin{tabular}{|c|c|c|c|c|c|c|c|}
\hline GALÇADO & $\begin{array}{c}\mathrm{N}^{0} \text { DE } \\
\text { POSTOS/ } \\
\text { ESTEIRA }\end{array}$ & $\begin{array}{c}\text { PRODUÇÃO } \\
\text { PROGRAMADA } \\
\text { (pares/dia) }\end{array}$ & $\begin{array}{l}\text { PRODUÇÃO } \\
\text { PROGRAMADA } \\
\text { (pares/10 min. } \\
\text { de operação) }\end{array}$ & $\begin{array}{c}k_{i j} \text { (pares/10 } \\
\text { minutos de } \\
\text { operação) }\end{array}$ & $k_{i j}^{r}$ & $\begin{array}{c}\boldsymbol{p}_{i j} \\
\text { (minutos) }\end{array}$ & $\begin{array}{c}r_{i j} \\
\text { (minutos) }\end{array}$ \\
\hline Calçado 8 & 2 & 1150 & 11 & 14,70 & 1,35 & 13 & 71,8 \\
\hline Calçado 9 & 2 & 520 & 5 & 15,10 & 3,07 & 5,1 & 42,6 \\
\hline Calçado 10 & 1 & 700 & 13 & 18,70 & 1,41 & 29,5 & 26,3 \\
\hline & & & \multicolumn{2}{|c|}{ Parâmetros médios } & 1,94 & 15,9 & 46,9 \\
\hline
\end{tabular}


a equipes de trabalhadores. O método proposto é fundamentado na modelagem dos dados de desempenho de trabalhadores submetidos a alterações freqüentes nos modelos de produtos através de curvas de aprendizagem. Para tanto, são apresentadas duas formas distintas de análise dos parâmetros das curvas de aprendizagem, de acordo com a duração estimada da corrida de produção. A utilização de procedimentos de clusterização permitiu a formação de famílias de produtos representativas e redu- ção na quantidade de dados a serem coletados para modelagem das curvas de aprendizagem.

Os dados de desempenho coletados foram utilizados para a avaliação de três modelos distintos de curvas de aprendizagem (hiperbólico de 3 parâmetros, exponencial de 3 parâmetros e tempo constante), segundo critérios de aderência aos dados e de capacidade preditiva. Os modelos avaliados apresentaram níveis de aderência inferiores aos obtidos por Mazur e Hastie (1978) e Nembhard e

Figura 3: Curvas geradas na execução de calçados pertencentes à família Difícil.
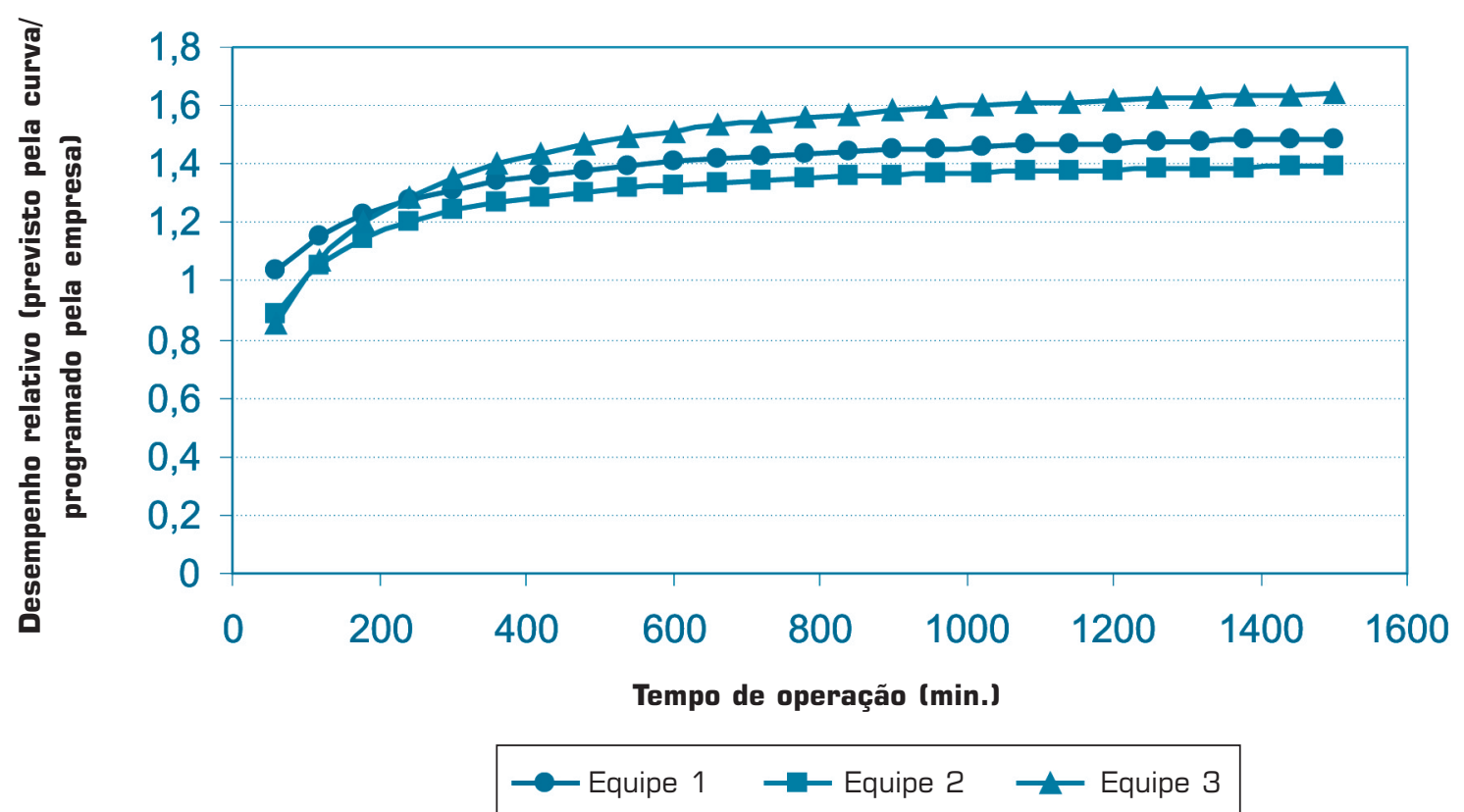

Tabela 8: Áreas geradas pelas três equipes na execução das operações relativas à família Difícil.

\begin{tabular}{|c|c|c|c|}
\hline \multirow{2}{*}{$\begin{array}{l}\text { TEMPO DE OPERAÇÃO } \\
\text { (min.) }\end{array}$} & \multicolumn{3}{|c|}{ ÁREA SOB A GURVA } \\
\hline & EQUIPE 1 & EQUIPE? & EQUIPE 3 \\
\hline 60 & 57 & 42 & 41 \\
\hline 120 & 122 & 101 & 99 \\
\hline 180 & 194 & 167 & 168 \\
\hline 240 & 269 & 238 & 243 \\
\hline 300 & 347 & 311 & 322 \\
\hline 360 & 426 & 387 & 404 \\
\hline 420 & 507 & 463 & 490 \\
\hline 480 & 590 & 541 & 577 \\
\hline 540 & 673 & 620 & 666 \\
\hline 600 & 757 & 700 & 756 \\
\hline 660 & 842 & 780 & 847 \\
\hline 720 & 927 & 860 & 940 \\
\hline
\end{tabular}


Quadro 1: Resumo das alocações sugeridas pelos dois métodos.

\begin{tabular}{|c|c|c|}
\hline \multirow[t]{2}{*}{ FAMÍLIA } & \multicolumn{2}{|c|}{ MÉTODO DE ALOGAÇÃO } \\
\hline & REGRESSÃO & INTEGRAÇÃO \\
\hline Fácil & Equipe 3 & Equipe 3 \\
\hline Média & Equipe 3 & Equipe 1 \\
\hline Difícil & Equipe 1 & $\begin{array}{l}\mathrm{t}_{\text {duração }}<600 \mathrm{mim} \text { - Equipe } 1 \\
\mathrm{t}_{\text {duração }}<600 \mathrm{mim}-\text { Equipe } 3\end{array}$ \\
\hline
\end{tabular}

Uzumeri (2000). Tal discrepância pode ser associada às condições de realização dos experimentos, principalmente em relação ao método de coleta de dados e à natureza das operações avaliadas. A capacidade preditiva dos modelos, entretanto, apresentou resultados satisfatórios quando avaliada em uma amostra de validação. O modelo hiperbólico de 3 parâmetros apresentou os melhores resultados de adequação aos dados, sendo utilizado para as etapas seguintes propostas pela metodologia.

A interpretação dos parâmetros das curvas de aprendizagem através de duas formas distintas (análise isolada do parâmetro $r_{i j}$ e integração das curvas de aprendiza- gem) permitiu a alocação de produtos a equipes de acordo com a duração da corrida de produção. A regressão mostrou-se adequada para a alocação de produtos a equipes em corridas de produção de curta duração, além de permitir a análise da influência de cada variável independente (equipe de trabalhadores, família de produto e interação equipe versus família) no processo de aprendizado dos trabalhadores. A integração, por sua vez, mostrou-se mais robusta que a regressão para fins de alocação, podendo ser utilizada em corridas de durações distintas mediante a comparação das áreas geradas pelas curvas de aprendizagem.

\section{Artigo recebido em 19/07/2004 Aprovado para publicação em 23/05/2005}

\section{- Referências Bibliográficas}

DA SILVEIRA, G.; BORESTEIN, D. FOGLIATTO, F. S. Mass Customization: Literature Review and Research Direction. International Journal of Production Economics, v. 72, p. 1-13, 2001

DE RON, A.J. Sustainable Production: the Ultimate Result of a Continuous Improvement. International Journal of Production Economics, v. 56-57, p. 99-110, 1998.

HAIR, J. F. JR.; ANDERSON, R.E.; TATHAM, R.L.; BLACK, W.C. Multivariate Data Analysis with Readings. 4. ed. New Jersey: Prentice-Hall Inc., 1995.
JOBSON, J. D. Applied Multivariate Data Analysis, Volume II: Categorical and Multivariate Methods. New York: Springer-Verlag, 1992.

MAZUR, J.E.; HASTIE, R. Learning as Accumulation: a Reexamination of the Learning Curve. Psychological Bulletin, v. 85 , n. 6 , p. $1256-1274,1978$.

NAIM, M.M.; TOWILL, D.R. An Engineering Approach to LSE Modeling of Experience Curves in the Electricity Supply Industry. International Journal of Forecasting, v. 6, p. 549-556, 1990.
NEMBHARD, D.A.; UZUMERI, M.V. An Individual-Based Description of Learning within an Organization. IEEE Transactions on Engineering Management, v. 47, n. 3, p. 370-378, 2000.

TEPLITZ, C.J. The Learning Curve Deskbook: A reference Guide to Theory, Calculations and Applications. New York: Quorum Books, 1991.

TOWILL, D.R. Forecasting Learning Curves. International Journal of Forecasting, v. 6, p. 25-38, 1990.
UZUMERI, M.; NEMBHARD, D. A Population of Learners: A New Way to Measure Organizational Learning. Journal of Operations Management, $\mathrm{v}$. 16, p. 515-528, 1998.

WRIGHT, T.P. Factors Affecting the Cost of Airplanes. Journal of the Aeronautical Sciences, v. 3, 1936.

YELLE, L.E. The Learning Curve: Historical Review and Comprehensive Survey. Decision Science, v. 10, 302328, 1979.

\section{- Sobre os autores}

\section{Michel José Anzanello}

M. Eng., pesquisador do LOPP/UFRGS

E-mail: michelja@producao.ufrgs.br

\section{Flávio Sanson Fogliatto}

$\mathrm{Ph}$. D., professor e pesquisador do LOPP/UFRGS

Departamento de Engenharia de Produção e Transportes - UFRGS

E-mail: ffogliatto@producao.ufrgs.br

Endereço: Praça Argentina, 9 - Sala LOPP - 90040-020 - Porto Alegre - RS

Fone: (51) 3316-4005 e (51) 3316-3491

Fax: (51) 3316-4007 